\title{
Las personas con discapacidad intelectual en el sistema penal penitenciario. ¿Un fracaso de las políticas de inclusión?*
}

\author{
Clarisa Ramos \\ Universidad de Alicante \\ clarisa.ramos@ua.es
}

Resumen: La situación de las personas con discapacidad intelectual que se encuentran insertas en el régimen penal penitenciario pone en evidencia la problemática social de un colectivo de alta vulnerabilidad que se encuentra en situación de exclusión social. El objetivo de este artículo es analizar las características de la población con discapacidad intelectual que se encuentra dentro del sistema penal penitenciario español, así como los procesos que determinaron su ingreso en él. El análisis se ha abordado desde una perspectiva cualitativa, respondiendo a una pregunta central que intenta saber si el ingreso de las personas en el sistema penal penitenciario supone un fracaso de las politicas de inclusión. Los datos se obtienen entre 2001 y 2010 y se centran en el trabajo realizado dentro de instituciones penitenciarias por la red FEAPS, incluido el ámbito de la comunidad autónoma de Cataluña (única con transferencia de competencias en cuestiones penitenciarias). Se realiza también una revisión de la bibliografía sobre la materia.

La falta de dispositivos que atiendan a las personas con discapacidad que manifiestan conductas tipificadas como delito pone de manifiesto el fracaso de las políticas de inclusión y prevención que se desarrollan en la sociedad. También queda de manifiesto una carencia de intervenciones integrales en las politicas relativas a inclusión social, que se analizan desde la discusión de resultados.

Palabras clave: discapacidad intelectual, sistema penal penitenciario, procesos exclusógenos.

\footnotetext{
* Este artículo fue presentado como ponencia en el IV Congreso REPS «Las políticas sociales entre crisis y post-crisis», Alcalá de Henares, 6 y 7 de junio de 2013.
} 
People with intellectual disabilities in the criminal justice prison system: A failure of inclusion policies?

Abstract: The situation of people with intellectual disabilities who are affected by the prison system highlights the social problems of a highly vulnerable and socially excluded group. The aim of this paper is to analyze the characteristics of the population with intellectual disability residing within the Spanish prison system, and the processes that caused them to enter the system. The analysis takes a qualitative approach that focuses on the central question of whether these individuals are entering the prison system due to a failure of inclusion policies. It also reviews the relevant literature. Data were obtained from research carried out in prisons between 2001 and 2010 by the Spanish Confederation of Organizations working for People with Intellectual Disability (FEAPS) and include data from the autonomous region of Catalonia (the only region with competence over its prison system).

The lack of resources devoted to people with disabilities who show behavior classified as criminal highlights the failure of the prevention and inclusion policies implemented by society. The results discussion also highlights and analyzes the lack of comprehensive intervention approaches regarding policies on social inclusion.

Keywords: intellectual disability, prison system, exclusion processes. 


\section{Introducción}

La inserción o reinserción social de las personas con discapacidad intelectual que pasan por el sistema penal penitenciario se encuentra vinculada a las oportunidades de incorporación a programas a los que, paradójicamente, solo llegan a acceder a partir de sus estancias en la cárcel. Decimos paradójicamente porque, por definición, la cárcel es la máxima expresión de la exclusión social y, sin embargo, existen casos en los que la supuesta «rehabilitación social» constituye antes un proceso de «habilitación», dado que, hasta su incorporación al sistema penal, algunas de estas personas carecían de recursos y apoyos para su integración en la sociedad.

Pero antes de poder hablar de la rehabilitación, la pregunta que surge es qué ocurrió con estas personas durante sus diferentes etapas vitales para que llegaran a encontrarse dentro del sistema penal penitenciario.

Al ser considerado un colectivo con mayor probabilidad de vulnerabilidad, podemos inferir que debieron pasar a lo largo del desarrollo de su vida por una serie de dispositivos de protección social. Cabe preguntarse por la actuación de las redes de apoyo, así como por las intervenciones de detección y prevención de situaciones de riesgo, y la influencia que pudieron tener para que una persona con discapacidad intelectual llegue al sistema penitenciario.

Nos preguntamos si la presencia de personas con discapacidad intelectual en el sistema penitenciario pone de manifiesto que estos dispositivos e intervenciones han estado ausentes o si, pese a haberse ejecutado, han fallado las estrategias de integración social que se plantearon.

Es desde este interrogante, surgido de la observación realizada durante el trabajo en este medio, del que partimos para formular la estrategia de investigación. Hay que conocer la actuación y posible influencia de redes asociativas, así como el papel de las redes de seguridad (safety nets), consideradas las últimas instancias de protección social que garantizan la posibilidad de acceder a recursos a aquellas personas que se encuentran en situación de mayor vulnerabilidad.

En este sentido, hemos querido describir los elementos que entran en juego en las situaciones delictivas y que nos permiten identificar los procesos de exclusión y de integración.

\section{Identificación del problema}

La situación de las personas con discapacidad intelectual en las cárceles está siendo objeto de particular atención en nuestro país en los últimos años, y también dentro del entorno europeo e internacional. Su presencia en el ámbito peniten- 
ciario español no pasa desapercibida, como puede comprobarse por la publicación de informes parlamentarios y documentos elaborados por el Ministerio del Interior.

En este mismo sentido, son muchos los trabajos que se vienen promoviendo desde el mundo de la investigación en discapacidad intelectual sobre las relaciones entre exclusión social, pobreza y discapacidad (Petersilia, 2000; FEAPS, 2006; Jones, 2007; Huete-García y Díaz-Velázquez, 2008; Williams, 2009; Clare y Gudjonsson, 2010; Ramos-Feijóo, 2011). Es por eso que nos parece que la cuestión presenta una notable actualidad y centralidad en el debate científico sobre la discapacidad intelectual, así como también en el debate sobre las políticas sociales que en el ámbito de la discapacidad se están desarrollando.

Asumiendo que la vulnerabilidad como concepto es relativa, ya que todos podemos ser vulnerables, es indudable que el colectivo de personas con discapacidad intelectual presenta un grado de vulnerabilidad más alto, en especial para las situaciones de delito en cualquiera de sus formas. Es por ello que consideramos que la presencia de dispositivos y la planificación de políticas sociales que atiendan a sus necesidades deben tener en cuenta estos supuestos.

En esta línea se han desarrollado diversas investigaciones, no solo teniendo en cuenta a la persona con discapacidad intelectual como autora, sino también como víctima de delito, y también se ha trabajado sobre la influencia de los problemas vinculados a la salud mental (Ayuso Mateos, Martorell, Novell, SalvadorCarulla, Tamarit, 2007).

La literatura existente sobre la cuestión nos acerca reiteradamente a la influencia de lo social en el delito, pero entendemos que se precisa también un conocimiento de las características individuales y sus posibles influencias en determinadas conductas. De este modo encontraremos recursos para la construcción de programas y políticas sociales que apoyen los procesos de integración social de las personas con discapacidad intelectual.

La historia del movimiento asociativo de la discapacidad intelectual, de sus características y orígenes, aporta información para entender algunos de los obstáculos encontrados a la hora de brindar atención e incorporar a las personas con discapacidad intelectual que han pasado por el sistema penal penitenciario a los diferentes recursos de atención. Lippman (1922) definía el concepto de estereotipo diciendo que «la mayoría de las veces no vemos primero y luego definimos, sino que definimos primero y luego vemos». El estigma de la cárcel conlleva a quien ha pasado por el sistema penal el cumplimiento de una nueva «pena» de exclusión social, esta vez no vinculada a una sentencia judicial, sino al estigma social. 
Siguiendo a Bayarri-Catalán (2010), autor directamente implicado en el mundo asociativo de la discapacidad, observamos que «los derechos nacen y se hacen cada día desde una ciudadanía activa y comprometida por parte de cada uno de nosotros, en el marco de nuestro compromiso social cotidiano, participando de todas aquellas dimensiones posibles que nos permiten avanzar en la construcción de una sociedad mejor» (Bayarri-Catalán, 2010:126).

En tabla 1 puede observarse cómo han ido evolucionando las líneas centrales de trabajo del movimiento asociativo FEAPS a lo largo de su historia. Se pone de manifiesto que el desarrollo de la autonomía personal y de la condición ciudadana ha pasado a representar una cuestión muy importante dentro de las líneas de trabajo y reflexión del movimiento asociativo.

Tabla 1. Cronología de prioridades temáticas en FEAPS

\begin{tabular}{|c|l|}
\hline \multicolumn{2}{|c|}{ Desarrollo del Movimiento Asociativo FEAPS } \\
\hline Década & Prioridades temáticas \\
\hline 60 & $\begin{array}{l}\text { Apoyo familia. } \\
\text { Escolarización. } \\
\text { Proceso de normalización. }\end{array}$ \\
\hline 70 & $\begin{array}{l}\text { Incorporación al mundo del trabajo. } \\
\text { Atención a las necesidades especiales de personas con discapacidad intelectual } \\
\text { grave. }\end{array}$ \\
\hline 80 & $\begin{array}{l}\text { Avances legislativos. } \\
\text { Acceso al empleo, incapacitación y tutela, curatelas, derechos sexuales (debate } \\
\text { sobre esterilización). }\end{array}$ \\
\hline 90 & $\begin{array}{l}\text { Sistema de apoyos: calidad de vida. } \\
\text { Autodefensores: autonomía personal. } \\
\text { Envejecimiento. } \\
\text { Papel de las familias. }\end{array}$ \\
\hline
\end{tabular}

Fuente: elaboración propia a partir de memoria histórica de FEAPS.

El movimiento asociativo se ha centrado en dar respuesta a las cuestiones vinculadas a las familias, la escolarización, la incorporación al mundo del trabajo y los procesos de normalización. Fue a partir de la década de 1990 cuando se trabajó sobre los sistemas de apoyo. Sin embargo, estos sistemas aún no se han desarrollado suficientemente para aquellos casos en que surgen problemas de trastornos de conducta vinculados a la salud mental, así como para la rehabilitación necesaria tras pasar por el sistema penal penitenciario. Esta circunstancia puede verse vinculada al hecho de que, en su génesis, este movimiento se nutre de la acción de las familias de clase media, que se encontraban con que no había una respuesta estructurada en las políticas sociales ni en los dispositivos de intervención sociosanitarios y educativos que atendieran las necesidades de las personas 
con discapacidad intelectual. Desde esa realidad se fueron construyendo los nuevos espacios de actuación y las respuestas desde la administración.

Continuando con la revisión de la literatura, seguimos a Abraam De Swaan (1992) en su planteamiento sobre las condiciones necesarias para que exista una conciencia de la necesidad de colectivizar las medidas de asistencia. Estas condiciones eran tres: por un lado, la incertidumbre, ya que no se sabe el momento ni la magnitud de la adversidad; por otro, también la incertidumbre en cuanto a la eficacia de los medios para contrarrestarla; y por último, la amplitud y el alcance de los efectos externos indirectos de las adversidades. En el marco de nuestro trabajo de investigación consideramos que las medidas de atención y apoyo a las personas con discapacidad intelectual comienzan a socializarse a partir de que existe una visualización de las necesidades de la discapacidad intelectual. En la medida en que éstas permanecían ocultas o dentro del ámbito de las soluciones brindadas por la solidaridad intrafamiliar, no era necesario que se dieran otro tipo de intervenciones. Es por eso que, si observamos la situación de las personas con discapacidad intelectual en las cárceles, hemos de tener en cuenta que constituyen un colectivo sobre el que resulta necesario realizar una intervención que dé respuesta a sus necesidades de atención y que ponga en evidencia la prioridad de desarrollar políticas preventivas.

Crear una mayor conciencia social sobre las necesidades de las personas con discapacidad mental que pasan por el sistema penal penitenciario en el conjunto de la ciudadanía significa promover una «conciencia de la generalización y la interdependencia que vincula a todos los miembros de una colectividad nacional y que va unida a un sentido abstracto de la responsabilidad que, si bien no impulsa la acción individual, exige que sea el Estado el que se ocupe, con fondos públicos, de dar protección a los necesitados en general» De Swaan (1992: 19).

Es importante asumir que cuando se habla del fracaso de las políticas sociales se entiende que las personas con discapacidad intelectual no están accediendo en igualdad de condiciones a las oportunidades, ni siquiera en aquellos países en los que se considera que existe un mayor nivel y calidad de vida. «Si la pobreza se define por la privación, ¿por qué las personas con discapacidades intelectuales y sus familias tienen tanta carencia? A escala mundial, todos los que participaron en este estudio dieron una respuesta similar: porque estamos excluidos - de los sistemas sociales, económicos y políticos que brindan oportunidades, recursos, reconocimiento y derechos» (Inclusion International, Informe «Oigan nuestras voces», 2006:5). Esto nos lleva a pensar que existe una percepción, por parte del propio colectivo, de exclusión en el acceso a determinados apoyos. Con la presencia en la cárcel de las personas con discapacidad intelectual, esta falta de apoyos especializados se hace aún más evidente. 
Partimos de la definición de exclusión social como un «proceso mediante el cual los individuos o grupos son total o parcialmente excluidos de una participación plena en la sociedad en la que viven» (European Foundation, 1995: 4). Interesa destacar la condición de proceso tanto de la exclusión como de la integración, dado que es en esta condición donde entendemos que se ancla la posibilidad de la intervención preventiva. El ingreso en el entorno penal penitenciario de una persona con discapacidad intelectual responde a un fracaso de las políticas dentro del proceso de integración y de socialización de esa persona, un proceso durante el cual han fracasado los dispositivos de apoyo necesarios para favorecer un desarrollo saludable.

\section{Metodología}

La elaboración del trabajo de investigación que sustenta este trabajo abordó diversos aspectos relativos a la situación de las personas con discapacidad intelectual que se encuentran en el sistema penal penitenciario. Se utilizaron datos cuantitativos y cualitativos, aunque el enfoque central del trabajo partió de una perspectiva cualitativa basada en la teoría fundamentada. Las estrategias de investigación se orientaron a responder las siguientes preguntas.

- ¿Qué aspectos influyen en las personas con discapacidad intelectual para que se vean inmersas en situaciones tipificadas como delito?

- ¿Cómo influyen la presencia o ausencia de sistemas de apoyo y de políticas inclusivas en que las personas con discapacidad intelectual ingresen en el sistema penal penitenciario?

- ¿Qué influencia tiene el papel de los agentes sociales, tales como organizaciones de asociaciones de personas con discapacidad, servicios sociales, sistema judicial, fuerzas de seguridad y sistema penitenciario?

Estas preguntas se abordaron a través de entrevistas y grupos de discusión, así como de fuentes documentales.

Dada la extensión del trabajo de investigación, hemos escogido el dato relativo a las políticas de inclusión social, pese a lo cual incorporamos un pequeño resumen en la tabla 2 donde se detallan las características de las tres macrocategorías que surgieron del microanálisis de las entrevistas a lo largo de la investigación.

Para este trabajo nos centraremos en la tercera, relativa a los procesos de exclusión y de integración, como forma de constatar nuestro interrogante de si la presencia de las personas con discapacidad intelectual en el sistema penal penitenciario puede considerarse resultado del fracaso de las políticas de inclusión social. 
Tabla 2. Características de las macrocategorías

\begin{tabular}{|l|l|l|}
\hline \multicolumn{1}{|c|}{ Macrocategorías } & \multicolumn{1}{|c|}{ Descripción de las macrocategorías } & \multicolumn{1}{c|}{ Observaciones (subcategorías) } \\
\hline $\begin{array}{l}\text { Personas con } \\
\text { discapacidad } \\
\text { intelectual }\end{array}$ & $\begin{array}{l}\text { Las personas con discapacidad intelectual } \\
\text { son el eje central de los argumentos, no se } \\
\text { habla de la cárcel en sí sino de las personas } \\
\text { con discapacidad que han llegado a la cárcel. } \\
\text { Se describe su realidad. }\end{array}$ & $\begin{array}{l}\text { Las subcategorías se relacionan con } \\
\text { las características personales, entorno } \\
\text { familiar, el diagnóstico y detección de } \\
\text { la discapacidad intelectual, patologías } \\
\text { asociadas. }\end{array}$ \\
\hline $\begin{array}{l}\text { Sistema penal } \\
\text { penitenciario }\end{array}$ & $\begin{array}{l}\text { El contexto en el que se desarrolla la investi- } \\
\text { gación entronca directamente con el sistema } \\
\text { penal penitenciario. } \\
\text { Los argumentos se vinculan a la comisión } \\
\text { de delito y a la presencia de las PDI en el } \\
\text { sistema penal penitenciario. }\end{array}$ & $\begin{array}{l}\text { Las subcategorías permiten un conoci- } \\
\text { miento más pormenorizado de la situa- } \\
\text { ción de las personas con discapacidad } \\
\text { intelectual que se ven inmersas dentro } \\
\text { de procesos judiciales. }\end{array}$ \\
\hline $\begin{array}{l}\text { Procesos de exclu- } \\
\text { sión/integración }\end{array}$ & $\begin{array}{l}\text { Las preguntas de investigación son retroali- } \\
\text { mentadas por el marco teórico y de interven- } \\
\text { ción y nos llevan a identificar dentro de los } \\
\text { argumentos los procesos que desembocan } \\
\text { en las situaciones de exclusión (ingreso en } \\
\text { la cárcel). }\end{array}$ & $\begin{array}{l}\text { Las subcategorías analizan la integración } \\
\text { social, tanto en el caso de que la misma } \\
\text { haya fracasado y se dé la exclusión, como } \\
\text { si el ingreso en la cárcel ha servido para } \\
\text { el proceso de integración. }\end{array}$ \\
\hline
\end{tabular}

Fuente: elaboración propia sobre investigación: La discapacidad intelectual en el ámbito penal penitenciario. Procesos de exclusión y de integración (Ramos Feijóo, 2010).

Centrándonos en los resultados de la macrocategoría relativa a los procesos de exclusión/integración, se identificaron posteriormente cinco subcategorías, que se desarrollan a continuación.

\subsection{La cárcel como fenómeno de exclusión, la cárcel como recurso de integración}

De la investigación surge como resultado la construcción de la cárcel como recurso de integración para aquellos casos en que no se ha podido encontrar un centro adecuado. Las personas entrevistadas perciben los centros penitenciarios como un recurso residencial ante la falta de un dispositivo más adecuado que, según se manifiesta, debiera estar en manos de los sistemas de protección social. Ahora bien, ante la carencia de políticas preventivas, se la identifica como último recurso del sistema. En este sentido, parece comprobarse que la afirmación de Wacquant (2000) sobre la existencia de una gestión policial de la pobreza podría parafrasearse en la existencia de una gestión policial de la discapacidad, fundamentalmente en aquellos casos en que la discapacidad intelectual pueda tener matices de conflictividad. No se pretende dar un cariz de control policial específico, sino que se lo emplea como una metáfora de la falta de una adecuada intervención social que termina trayendo como consecuencia un control social personalizado en las fuerzas de seguridad, entendiendo dentro de las mismas todo el sistema penal penitenciario (tanto las fuerzas y cuerpos de seguridad como el sistema judicial). 
Ante el retroceso de los sistemas de protección se observa que hay personas que, hasta su ingreso en el sistema penitenciario, en su mayoría no habían sido identificadas por esos sistemas, y que, si lo habían hecho, era desde una perspectiva de labeling, es decir, como «casos crónicos»o «casos de largo recorrido», con la consiguiente estigmatización que ello conlleva.

\subsection{El centro penitenciario como recurso social de institución cerrada}

Procesos como el de la reforma psiquiátrica son considerados por algunos de los entrevistados como un factor detonante de la consolidación de las instituciones penitenciarias como un recurso social. Las personas entrevistadas destacan que no se trata de una crítica a la reforma psiquiátrica en sus fundamentos, sino a la falta de recursos que los desarrollen e implementen para aquellas personas que presentan necesidades de apoyo específico y continuado para su integración social. Estas carencias se identifican en las entrevistas como un factor de fracaso de las políticas sociales, que se pone de manifiesto en la identificación del centro penitenciario como recurso social de institución cerrada.

En este sentido, el Hospital Psiquiátrico Penitenciario como ámbito de cumplimiento de medidas de seguridad alcanza a cubrir cuotas de protección que hace que algunos usuarios no deseen irse, ya que, aun privados de su libertad, se encuentran más protegidos dentro del ámbito cerrado. En el discurso se percibe que la intervención pospenitenciaria se presenta como un salto al vacío por la carencia de intervenciones que tengan un adecuado grado de contención, ya sea en lo familiar o institucional, para el exinterno. No se trata de carencia de protocolos, ya que existe un acuerdo a la hora de definir la importancia de la intervención preventiva, de la preparación para la salida, tanto por parte de profesionales del tercer sector implicado como de los servicios sociosanitarios y de la propia institución penitenciaria. Se define la carencia de una visión integral a la vez que individualizada en la intervención.

La intervención en el centro penitenciario el interno la percibe como un recurso de protección, es allí donde se siente integrado. Se recogen varios testimonios de casos en los que los internos quieren regresar a los centros una vez cumplida la medida de seguridad o la pena, ya que consideran que allí se encontraban en mejores condiciones. Nuevamente se observa el vacío del tratamiento y seguimiento pospenitenciario. La cita pone en evidencia la falta de un referente en el «proceso de inserción social», ya que, si bien puede comenzar dentro del sistema penal penitenciario, no tiene en él su fin, sino en todo caso una preparación para la lógica de la vida en libertad. 
Queda reflejada, así pues, la existencia de una problemática ante la falta de recursos residenciales, donde el centro penitenciario está considerado como un recurso social. La definición de que las instituciones totales «son primas hermanas» parece reflejar de manera muy gráfica la inexistencia de dispositivos que aborden la problemática de las personas con discapacidad intelectual. Se destaca la referencia a la dificultad por parte de las fuerzas de seguridad para poder diferenciar entre un acto torpe y un delito. En este caso el acto torpe podría ser constitutivo de la elaboración de un acta policial, mientras que, si de una discusión se elabora un atestado, se sabe que ese caso terminará ante un juez, y que probablemente la persona con discapacidad intelectual terminará afrontando un proceso judicial que puede desembocar en una medida de seguridad o en una pena. Esta constatación permite que se observe en los argumentos la presencia de una demanda de formación adicional, tanto en las fuerzas y cuerpos de seguridad como en el propio personal de los servicios sociales y sanitarios.

El recurso residencial cerrado se define como una solución que no se contempla como negativa para un número pequeño de los casos que no encuentran otro tipo de recurso alternativo que les permita desarrollar una vida autónoma y normalizada. En estos supuestos, las necesidades de apoyo y contención no podrían ser satisfechas por el ámbito familiar ni tampoco por un contexto institucional comunitario. Nuevamente recupera centralidad en el discurso la discontinuidad del tratamiento una vez cumplida la medida de seguridad o la pena.

Se detecta como problema la ruptura del itinerario terapéutico una vez cumplida la medida o pena. Es decir, al parecer, dentro del centro penitenciario, las personas que entran en los programas especializados de FEAPS reciben una atención individualizada que no continúa cuando salen. En el discurso se evidencia también que no se ha logrado una incorporación de la familia al tratamiento. En el cuaderno de campo se señala que, al preguntarse por el ámbito del tratamiento familiar, existe el argumento de la dificultad que acarrea la distancia desde los centros penitenciarios a los domicilios de las familias, lo que hace inviable, según la información obtenida, una intervención familiar satisfactoria.

\subsection{El estigma de la prisión}

En las entrevistas se reflejan las dificultades añadidas a los itinerarios de integración que provoca el llamado «estigma de la prisión». Se destaca que, dentro de las propias asociaciones integrantes de la red FEAPS, existen resistencias para aceptar a personas que hayan pasado previamente por el sistema penal penitenciario, pese a que sean sus propios equipos técnicos los que hayan trabajado con estos casos y soliciten su incorporación a recursos normalizados. 
Tal y como se ha mencionado en la identificación del problema, este hecho obedece entre otras razones a las características del movimiento asociativo de la discapacidad intelectual, y demuestra la necesidad de realizar un proceso de sensibilización no solo en la sociedad en general, sino incluso en las propias asociaciones del sector.

En el discurso de los profesionales del mundo asociativo que intervienen en los programas vinculados al ámbito penal penitenciario se considera que, con los apoyos adecuados, no existen grandes diferencias en los perfiles de comportamiento de las personas con discapacidad intelectual que pasan por el sistema penal de los que pueden verse en los recursos habituales de los centros de la red FEAPS. Sin embargo, hay acuerdo en afirmar la necesidad de una doble estrategia que, por un lado, apunte a la sensibilización, y por otro, a una mayor especialización en la formación.

\subsection{Recursos para la integración social poscarcelaria o alternativas a la prisión}

La gestión se concibe personalizada; hay siempre una referencia a una figura de autoridad en el sistema administrativo que es la encargada de reclamar que se adjudique un recurso a la persona que sale de la cárcel.

En los discursos se manifiesta así mismo una falta de coordinación con los servicios sociales; al parecer, por los relatos, la «correa de transmisión» que podría favorecer el proceso no se encuentra convenientemente conectada.

Se identifican también argumentos que desplazan la falta de recursos hacia la falta de decisión para implementar esos recursos. La normativa avala el cumplimiento de las intervenciones. Desde el punto de vista administrativo atribuye a las comunidades autónomas la responsabilidad de la asignación de un recurso pospenitenciario para las personas con discapacidad intelectual, o bien con un diagnóstico de enfermedad mental, que requieren de un sistema de apoyo para su integración social. Sin embargo, su puesta en práctica parece encontrar muchas trabas operativas.

Observamos lo que definimos como la paradoja de los procesos de integración-exclusión, es decir, una vez que comienza la intervención de los sistemas de protección social se produce una sucesión de derivaciones definida como «la dinámica del sistema de reinserción», que en el discurso se relaciona con la marginalidad. La reinserción se vincula a las listas de espera para recursos residenciales, es decir, que la argumentación nos conduce a ver que la salida para la integración social de las personas con discapacidad intelectual cuando egresan de un centro penitenciario pasa por un recurso residencial, porque en un alto porcentaje la 
realidad familiar no se encuentra preparada para darles la contención adecuada. Se destaca aquí también que, en muchos casos, aun queriendo, las familias carecen de los apoyos para poder hacerse cargo de las personas con discapacidad intelectual, fundamentalmente en los casos en que se encuentra asociado algún trastorno de conducta.

\subsection{Trabajo de rehabilitación/habilitación}

De las entrevistas se desprende que los subsistemas de bienestar y el tercer sector no alcanzan a ejecutar suficientes acciones de intervención preventivas de las situaciones de delito en las personas con discapacidad intelectual de los entornos socialmente más desfavorecidos.

Para encontrar respuesta a esta cuestión entendimos necesario:

\subsubsection{Conocer las formas de intervención del mundo asociativo en esta problemática}

El papel de las organizaciones no gubernamentales aparece como de una gran importancia pero con un alcance insuficiente, toda vez que se sigue manteniendo el corte entre la integración institucional y la posterior salida a la vida social. Así mismo, no se detectan políticas preventivas, o políticas de intervención flexible, que atiendan las necesidades de apoyo intensivo que algunas de estas personas necesitan. En algunos casos se da continuidad al proceso de integración social, pero no como un protocolo de intervención habitual sino como respuesta a las circunstancias en las que se hace totalmente viable, tanto por las características de la persona como por la existencia del recurso. Esta no es la situación habitual principalmente en aquellos casos en los que la discapacidad intelectual coincide con algún trastorno mental.

3.5.2 Identificar si los servicios sociales establecen alguna linea de trabajo especial para esta problemática, o si identifican la realidad de las personas con discapacidad intelectual que se encuentran dentro del sistema penal penitenciario como una línea de trabajo para la integración social de las personas con discapacidad. Identificar si se realizan intervenciones preventivas de situaciones de riesgo con las personas con discapacidad intelectual.

En cuanto al papel de los servicios sociales, se observa una cierta desconexión entre los mismos y el sistema penal penitenciario, e incluso el judicial. Este hecho ha podido observarse no solo en los casos de las personas con discapacidad intelectual en las cárceles, sino incluso en el estadio previo, que también fue analizado 
a través de entrevistas a expertos en el área de infancia. Allí pudo comprobarse que, tanto en protección como en reforma, existe una insuficiente coordinación con los equipos de salud mental y de atención a la discapacidad. Parece que resulta evidente, de la información obtenida, que más allá de los protocolos no se ha generalizado la presencia de una metodología de trabajo continuada, que pareciera coincidir mejor con la idea de la gestión de casos como instancia superadora de las especializaciones. Este aspecto no parece contemplarse en las políticas de servicios sociales orientados hacia la discapacidad.

El trabajo realizado con las familias se presenta como insuficiente, tanto desde la red FEAPS como desde las Instituciones Penitenciarias. En algunos testimonios recogidos se apunta como una de las primeras dificultades la lejanía que suele existir entre los centros penitenciarios y los lugares de residencia de las familias. Pero en general no se ha identificado la existencia de un procedimiento para el trabajo social o psicoterapéutico de las familias en profundidad y con una continuidad que vaya más allá de la comunicación telefónica o de la entrevista diagnóstica. En todo caso se considera que, dadas las características de la atención que requiere esta población, la intervención familiar resulta insuficiente. Pero, al mismo tiempo, esta intervención familiar tampoco se ha identificado en las políticas sociales preventivas previas a la comisión de cualquier delito.

Teniendo en cuenta estos aspectos surge como pregunta el papel que juega la cárcel en los procesos de exclusión y de integración, y nos preguntamos si era posible que en algunos casos la cárcel fuera el lugar en que algunas personas con discapacidad intelectual toman contacto con programas que favorecen su integración social, dejando constancia expresa que hablamos de «in-tegración» y no de «re-integración».

Para ello resultó a su vez necesario:

3.5.3 Analizar los antecedentes de las personas con discapacidad intelectual que se encuentran en las cárceles, en lo relativo a su relación previa con el mundo asociativo y con programas de integración social. Conocer el discurso de las personas expertas en cuanto a los posibles factores causales que desembocaron en situaciones de delito.

De la información obtenida se deduce que, en muchos casos, las personas con discapacidad intelectual han percibido la institución penitenciaria como un ámbito contenedor donde pareciera encontrarse satisfacción a una serie de necesidades de subsistencia y sociabilidad; fundamentalmente en los casos en que la existencia previa carecía de elementos de contención. Así, en alguno de los casos ejemplificados como de integración, el planteamiento del propio sujeto es que, a 
partir de la incorporación a un programa de FEAPS, logra reorientar su vida, y de hecho, pese a que el delito cometido era grave e implicó la separación de su grupo familiar, paralelamente le facilitó la incorporación a una vida autónoma en un piso tutelado. Esta autopercepción aparece en el relato de algunas de las personas con discapacidad entrevistadas, y también de manera indirecta en la descripción que las personas expertas hacen al manifestar que son numerosos los ejemplos de personas que solicitan volver a las instituciones en las que estuvieron recluidas.

Se pone así de manifiesto un fracaso, o al menos una falta de especialización en la intervención desarrollada desde las políticas sociales que trabajan en la integración social.

\section{Conclusiones}

Estas circunstancias nos llevan a concluir que los procesos de integración están directamente vinculados con las oportunidades que se les brindan a las personas con discapacidad intelectual que han cometido un delito para que se incorporen a un itinerario diferente al que las pudo llevar a la cárcel.

En la medida en que se dan una serie de apoyos individualizados y se hace un seguimiento de cada persona atendiendo a sus necesidades específicas, puede estimarse como posible el proceso de integración dentro de unas expectativas de calidad de vida. Esto supone comprender que, en algunas circunstancias, es necesaria la atención psiquiátrica, la orientación laboral, e incluso en los casos en los que existe un núcleo familiar, será necesaria la asistencia al mismo, ya que la sola disposición para hacerse cargo de la persona con discapacidad intelectual no presupone el que se logre una interacción positiva y favorable para la integración dentro de una comunidad.

Teniendo en cuenta los aspectos inacabados de la Reforma Psiquiátrica española confrontamos los resultados de las entrevistas con la opinión de Moretón Sanz (2011): «el modelo y la propia ausencia de patrones para la atención de las personas con enfermedad mental o discapacidad intelectual evidencia una evolución normativa que debería dirigirse con más entusiasmo hacia los derechos de los pacientes, y ampliarse en el denominado cuarto pilar del Estado social [... ]». Esta cita nos vincula con la falta de recursos especializados, o al menos con la inexistencia de una capacidad de intervenir de manera individualizada y flexible a la vez. Este hecho aparece, en los casos investigados, como una recurrente demanda de calidad.

Se observa la necesidad de poner en marcha recursos pensados desde el enfoque de especialización flexible que intervengan desde estructuras menos regula- 
das en una dimensión más cercana, más local. Estos dispositivos tienen una flexibilidad que les permite «hacer un diagnóstico rápido de un problema concreto y articular con agilidad una respuesta provisora específica» (Jaraiz, 2011).

Existe en los itinerarios de las personas con discapacidad intelectual que llegan a la cárcel una similitud que pareciera darse en la falta de apoyos suficientes tanto en el entorno familiar como en la red social, entendiendo dentro de la misma tanto a los servicios sociosanitarios como a las asociaciones específicas del sector.

En este sentido nos parece importante recordar que los caminos de exclusión no están vinculados a variables exclusivamente socioeconómicas sino, antes bien, a factores como la falta de alcance de los dispositivos de protección social a las personas que salen del circuito habitual. Los casos que hemos analizados habían pasado de forma mayoritaria por Servicios Sociales pero habían sido desestimadas por la «carencia del recurso apropiado». Más allá de los dispositivos creados por los sistemas de protección, debe verse las necesidades de cada persona, y en este sentido entendemos que es donde se daría una «filtración» de las mallas de seguridad. Cuando alguien no responde a las características para la atención definidas en la norma, parece observarse que los circuitos conducen a su expulsión del sistema, por lo que, por lo general, concluyen en la máxima expresión de la exclusión, que es el sistema penal penitenciario.

Las instituciones penitenciarias aparecen consolidadas en las entrevistas como un «recurso social» ante la carencia de recursos. Desde quienes consideran que, hasta tanto no se encuentren otras salidas, lo importante es mejorar la calidad de la asistencia a las personas con discapacidad en el sistema penitenciario, hasta a quienes ven en esta situación un reflejo de cierta «hipocresía social» por la que la sociedad desea que se atienda bien y se garantice la calidad de vida de las personas con discapacidad que presentan problemas de conducta, pero en realidad prefieren que esta atención se les brinde en condiciones lo más controladas posibles, es decir, recurriendo al encierro.

Del mismo modo, no se han detectado líneas de políticas de intervención comunitarias para evitar la comisión de delitos que hayan puesto para ello particular interés en las acciones sociales y sanitarias de carácter preventivo, en las que se incluyen los servicios de salud con especial atención en los de salud mental, los servicios educativos, los servicios sociales y el movimiento asociativo.

En este sentido, llama la atención también el hecho de que en numerosas entrevistas se destaca el deseo de las personas con discapacidad de seguir permaneciendo dentro de las Instituciones Penitenciarias. A nuestro entender esto define un problema de «cortocircuito» en el proceso de integración, dado que si una per- 
sona llega a verbalizar que cometerá otro delito para volver a la cárcel es porque el proceso de preparación para la vida social no se ha completado, o que al menos no se la ha preparado para la vida en libertad. Este punto sin duda constituye uno de los desafíos más grandes del tratamiento penitenciario, que se hace evidente no solo en las personas con discapacidad, sino con la población reclusa en general.

De esta observación podemos inferir que hacen falta mayores dispositivos de preparación de ese proceso que llamamos de «integración», dado que, de lo contrario, podría surgir la paradoja de que estuviéramos trabajando para integrar en la exclusión. En este sentido encontramos coincidencias con estudios comparativos realizados en la materia, aunque en el ámbito de las mujeres en prisión, como es el proyecto MIP, donde se concluyó que existe una contradicción entre los principios y la práctica de la justicia criminal y social, ya que, pese al énfasis que se da a la función de integración social, resulta muy difícil conciliar la preparación para la integración social con el sistema penitenciario y su realidad, que por definición produce un agravamiento de la exclusión social que se hace más evidente en los grupos más vulnerables ${ }^{1}$.

En otros casos, el ingreso en prisión ha significado una incorporación a dispositivos de mayor protección social; son aquellos en los que, a través de los programas FEAPS y con el apoyo de recursos personalizados, se ha logrado una excarcelación y una integración en algún dispositivo especializado (piso tutelado, centro especializado). Estos casos habían pasado desapercibidos hasta el momento de su ingreso en prisión por los dispositivos de protección, o habían resultado «inadaptables» a los mismos. Suele ser el caso de las personas que presentan un diagnóstico dual, o de aquellos que provienen de situaciones de exclusión social previa al ingreso en la cárcel. El aporte del dispositivo penitenciario es que visualiza el problema y las necesidades de esta persona, y si existe un equipo que pueda realizar el seguimiento se logrará con mayor o menor acierto un recurso adaptado a su capacidad.

A vueltas con la prevención y sus agentes, en las entrevistas con personas expertas y la observación participante se infiere que la actividad preventiva es muy generalista, no llega de manera específica a las posibles causas generadoras de situaciones delictivas. Se identifica alguna experiencia preventiva, como la de un proyecto europeo desarrollado por la Fundación Paz y Bien en Sevilla, que está considerada como una experiencia pionera que los Servicios Sociales de la Junta

1 Informe proyecto MIP, Noelia Igareda (2005). SURT Mujeres, Integración y Prisión, Aurea Editores, disponible también en la página <web www.surt.org >. «Mujeres, integración y prisión. Un análisis de los procesos de integración sociolaboral de las mujeres presas en Europa». Proyecto Europeo bajo el V Programa Marco «Mejora de la base de conocimientos socioeconómicos». 
de Andalucía han recogido, pero que como tal no llega a consolidar una pauta de trabajo preventivo institucionalizada.

De las entrevistas mantenidas se deduce que, dentro del movimiento asociativo, existen aún reticencias para el trabajo con personas con discapacidad que presentan conductas delictivas, en parte por prejuicios y por temor, y en un alto porcentaje por desconocimiento de las intervenciones que se podrían realizar, tanto de manera preventiva como para evitar la reincidencia o para contribuir al proceso de reinserción social.

Se observa una escasa coordinación entre los ámbitos de infancia, juventud y discapacidad en lo relacionado con problemas de conducta. Es decir, no existe un trabajo de red en el que se puedan vincular las asociaciones que trabajan en el campo de las medidas judiciales juveniles con el campo de la salud mental y la discapacidad intelectual. Es cierto que, al menos en este último ámbito, la relevancia estadística parece ser escasa, pero justamente por ello podría trabajarse más individualizadamente el aspecto preventivo.

\section{1 Ámbitos para el planteamiento de propuestas}

En el planteamiento metodológico establecimos como metas realizar propuestas en los cuatro siguientes ámbitos:

- Proponer posibles actuaciones desde los servicios sociales para la detección de situaciones de riesgo en el ámbito de las personas con discapacidad intelectual.

- Analizar la viabilidad de propuestas de prevención de las situaciones de delito en las que se encuentran personas con discapacidad intelectual.

- Proponer medidas de mediación para evitar el acceso a las instituciones penitenciarias de las personas con discapacidad intelectual.

- Conocer prácticas realizadas en otros países que puedan ser replicadas en nuestro país.

Tras las conclusiones de la investigación pensamos que sería de utilidad desarrollar una línea de investigación en el ámbito de los servicios sociales para la definición de estrategias de intervención que favorezcan la detección de situaciones de riesgo para las personas con discapacidad intelectual. En este sentido, consideramos necesario rescatar experiencias como las ya desarrolladas a través de experiencias piloto como el programa Horizon Alas, o el Alerta, pero llevando las ideas desarrolladas como buenas prácticas a la intervención cotidiana.

La calidad de vida y el desarrollo del sistema de apoyo son ejes centrales del discurso del movimiento asociativo FEAPS; entendemos, por tanto, que estos 
dos ejes deben aplicarse a los aspectos preventivos no solo con los sectores considerados de mayor vulnerabilidad por su situación socioeconómica, sino al conjunto de las personas con discapacidad intelectual.

Los servicios sociales tienen una grave dificultad para las tareas preventivas, ya que se ha consolidado una tendencia de atención a la demanda que dificulta la detección previa de aquellos casos que, precisamente por su mayor complejidad, escapan a los sistemas de protección consolidados. En esta misma línea aparece como una necesidad acuciante la de establecer metodologías coordinadas de intervención, y reiteramos la conveniencia de los sistemas de gestión de casos, ya que en situaciones en las que existen varios aspectos sobre los cuales es preciso trabajar resulta necesaria una coordinación que sobrepasa el exclusivo cumplimiento de un protocolo.

La ruptura comprobada en los procesos de integración social que se da entre el cumplimiento de una pena o medida de seguridad y la incorporación a la vida comunitaria nos da cuenta de una esquizofrenia en los discursos. Resulta imprescindible, en este sentido, que cuando una persona con discapacidad ha cometido un delito cuente con alguien que gestione su caso, que resulte una figura tutora $\mathrm{o}$ como prefiera llamársele, pero es evidente que, de cara a la prevención, es necesario un proceso de acompañamiento que en la actualidad no se está dando.

En el ámbito de las propuestas sobre figuras mediadoras que eviten el ingreso de las personas con discapacidad en las cárceles, entendemos que, en primer lugar, existe una carencia de recursos humanos suficientes formados en el área de discapacidad intelectual, tanto en las profesiones del ámbito forense (psicología, medicina, psiquiatría, trabajo social) como en las de las esferas jurídica y social. Una primera línea de propuestas irá encaminada a la necesidad de capacitar, tanto a nivel de grado como de posgrado, a las profesiones directamente implicadas en los procesos de integración social, enmarcando esta formación en un proceso de sensibilización más amplio.

Las experiencias desarrolladas en otros países, de las que también contamos con algunas iniciativas en España, en el ámbito de los convenios con la policía nos parecen ciertamente una fórmula acertada para evitar la consideración de delito en aquellos casos que pudieran ser solventados como hechos torpes sin llegar a precisar de la intervención del ámbito penal.

Del análisis de las entrevistas, así como de la explotación de los datos y la información pormenorizada que sobre la población hemos obtenido, se observa que el sistema penitenciario recibe un colectivo de personas cuyo tratamiento debiera realizarse desde sistemas de atención más normalizados y más especializados para responder a sus necesidades y potenciar sus capacidades. Entendemos 
que ese sería un proceso de integración social más digno y coherente con la calidad de vida que toda persona merece.

Sin embargo, quizás como consecuencia paradójica del proceso de normalización, podría entenderse hasta lógico que existan personas con discapacidad intelectual en el ámbito penal penitenciario, pero es evidente que esta circunstancia podría haber sido evitada. Entendemos que una forma de prevenir estas situaciones tiene relación directa con una adecuada detección de las situaciones de riesgo en que se encuentran las personas de estos colectivos, y que exceden las situaciones de pobreza económica. Por ende faltan, o no están suficientemente aprovechados, los dispositivos sociosanitarios que contribuyan a la integración social efectiva de las personas con discapacidad intelectual que puedan presentar perfiles conflictivos.

Insistimos en la necesidad de establecer sistemas de intervención integral de los casos en que se encuentren situaciones de riesgo en que puedan involucrarse todas las administraciones con competencias en la integración social. En este sentido, aportan interesantes expectativas los procesos de trabajo comunitario que se están presentando en las propuestas de salud mental de comunidades autónomas como el Principado de Asturias, así como también en lo que se desprende del contenido aprobado en la Ley de Barrios de la comunidad autónoma de Extremadura. Esta normativa tiene como objetivo crear un marco legal para coordinar las políticas de la Administración regional y local, así como de entidades de iniciativa social, para favorecer la inserción social y laboral de las personas en situación o riesgo de exclusión social que residan en zonas vulnerables; y propiciar una actuación integral y más efectiva, que englobe todo el conjunto de acciones de carácter urbanístico, de vivienda, económico y social que mejore la atención a estos ciudadanos. Se trata de una normativa autonómica promovida por la Consejería de Igualdad y Empleo que plantea abordar las problemáticas de forma integral desde las siguientes áreas: servicios sociales, mujer, sanidad, educación, urbanismo y vivienda, formación y empleo, comercio y empresas, seguridad, participación social, ocio y juventud.

Se continúa trabajando en líneas de investigación para profundizar los sistemas de identificación y diagnóstico de las personas afectadas para determinar la dimensión cuantitativa y cualitativa del colectivo a atender dentro del sistema penitenciario, extendiendo la valoración a los sistemas de justicia juvenil.

Se ve conveniente evaluar las posibles estrategias mediadoras para evitar el ingreso de las personas con discapacidad intelectual en dispositivos del sistema penal. En este caso se citan experiencias como las que se desarrollan, entre otros países, en EE.UU. mediante los Mental Health Court o los Problem-Solving 
Courts, que funcionan como tribunales comunitarios cuyo objetivo es resolver los problemas vinculados a lo que definimos como «hechos torpes o conflictos de baja intensidad».

Entendemos que, en esta línea, podría analizarse desde una visión de políticas de inclusión que intervengan desde la perspectiva preventiva la problemática delictiva en el área de la discapacidad y la enfermedad mental, ya que carece de sentido que estas personas acaben siendo atendidas por el sistema penitenciario, que, lejos de resolver los procesos de exclusión social, los agudiza.

\section{Bibliografía}

Ayuso-Mateos, J. L.; Martorell, A.; Novell, R.; Salvador-Carulla, L.; Tamarit, J. (2007). Discapacidad intelectual y salud mental. Guía práctica. Madrid: Consejería de Familia y Asuntos Sociales.

Bayarri Catalán, V. (2010). «La regulación pendiente de los derechos sociales a la inclusión en la comunidad de personas con discapacidad», en PÉrez Bueno L. C. (dir.) Discapacidad, tercer sector e inclusión social. Estudios en homenaje a Paulino Azúa. Ediciones Cinca Fundación Derecho y Discapacidad y CERMI. Colección CERMI.

Clare, I. C. H.; Gudjonsson, G. H. (1995). "The vulnerability of suspects with intellectual disabilities during police interviews: a review and experimental study of decision-making», Mental Handicap Research, 8: 110-128. doi: 10.1111/j.1468-3148.1995.tb00149.x

De Swann, A. (1982). A cargo del Estado. Barcelona: Ediciones Pomares Corredor.

European Foundation for the Improvement of Living and Working Conditions (1995). Public Welfare Services and Social Exclusion: The Development of Consumer Oriented Initiatives in the European Union. Dublín.

Huete-García, A.; Díaz Velazquez, E. (2008). «Personas con discapacidad afectadas por el sistema penal-penitenciario en España», Revista Zerbitzuan, 44.

InClusión InTERnACional (2006). Oigan nuestras voces: Un informe global. Las personas con discapacidad intelectual y sus familias hablan claro sobre la pobreza y la exclusión.

Jaraíz Arroyo, G. (2011). Intervención social, barrio y servicios sociales comunitarios. Madrid: Editorial Foessa,

Lippmann, W. (1922). Public Opinion. Nueva York: Macmillan.

Moretón SAnz, M. ${ }^{a}$ F. (2011) «La inacabada reforma psiquiátrica española: cuestiones sobre internamientos psiquiátricos no voluntarios y la ausencia 
de control judicial en los ingresos geriátricos», Comunitania. Revista Internacional de Trabajo Social y Ciencias Sociales, enero, n. 1, pp. 69-87. En línea: <http://dx.doi.org/10.5944/comunitania.1.4>

Petersilia, J. (2000). «Doing justice? Criminal offenders with developmental disabilities», CPRC Brief, 12 (4), California Policy Research Center, University of California.

Ramos Feijóo, C. (2011). «Las personas con discapacidad intelectual en el sistema penal-penitenciario. Algunas paradojas de la integración en la exclusión». Boletín del Real Patronato sobre Discapacidad, 8-14.

VV.AA. (2006). Guía de intervención para personas con discapacidad intelectual afectas por el régimen penal penitenciario. Madrid: FEAPS y Obra Social Caja Madrid.

Williams, C. (2009) «Scant justice». Community Care, 26-27. 